\title{
Unexpected clinical outcomes following the implementation of a standardised order set for hepatic encephalopathy
}

\author{
Mandip KC (D) , ${ }^{1}$ Andrew P J Olson, ${ }^{2,3}$ Qi Wang, ${ }^{4}$ Nicholas Lim (i) ${ }^{1}$
}

\begin{abstract}
To cite: $K C$ M, Olson APJ, Wang $Q$, et al. Unexpected clinical outcomes following the implementation of a standardised order set for hepatic encephalopathy. BMJ Open Gastro 2021;8:e000621. doi:10.1136/ bmjgast-2021-000621
\end{abstract}

Received 10 February 2021 Revised 14 March 2021 Accepted 27 March 2021

\section{Check for updates}

(C) Author(s) (or their employer(s)) 2021. Re-use permitted under CC BY-NC. No commercial re-use. See rights and permissions. Published by BMJ.

${ }^{1}$ Department of Medicine, Division of Gastroenterology, Hepatology and Nutrition, University of Minnesota Twin Cities, Minneapolis, Minnesota, USA

${ }^{2}$ Department of Medicine, Division of General Internal Medicine, University of Minnesota Twin Cities, Minneapolis, Minnesota, USA ${ }^{3}$ Department of Pediatrics, Division of Pediatric Hospital Medicine, University of Minnesota Twin Cities, Minneapolis, Minnesota, USA ${ }^{4}$ Clinical and Translational Science Institute, University of Minnesota Twin Cities, Minneapolis, Minnesota, USA

Correspondence to Dr Mandip KC;

kcxxx011@umn.edu

\begin{abstract}
Objective We evaluated the effect on clinical outcomes of implementing a standardised inpatient order set for patients admitted with hepatic encephalopathy (HE).

Methods A retrospective review of patients with cirrhosis admitted with HE. Hospital admissions for HE for which the electronic health record (EHR) order set was used were compared with admissions where the order set was not used. Primary outcome was length of hospital stay (LOS). Secondary outcomes were 30-day readmissions, inhospital complications, in-hospital and 90-day mortality. Results There were 341 patients with 980 admissions over the study period: 263 patients with 736 admissions where the order set was implemented, and 78 patients with 244 admissions where the order set was not implemented. Median LOS was 4 days (IQR 3-8) in the order set group compared with 3 days (IQR 2-7) $(p<0.001)$; incidence rate ratio $1.37(95 \% \mathrm{Cl} 1.20$ to 1.57), $p<0.001$. 30 -day readmissions rate was $56 \%$ in the order set group compared with $40 \%, p=0.01$; OR for readmission was $1.88(95 \% \mathrm{Cl} 1.04$ to 3.43$), \mathrm{p}=0.04$. Hypokalaemia occurred in $46 \%$ of admissions with order set use compared with $36 \%$, when the order set was not used; $p=0.003$, OR $1.72(95 \% \mathrm{Cl} 1.22$ to 2.43$), \mathrm{p}=0.002$. No significant differences were seen for in-hospital mortality and 90-day mortality.

Conclusion Implementation of an inpatient EHR order set for use in patients with HE was associated with unexpected clinical outcomes including increased LOS and readmissions. The convenience and advantages of standardisation of patient care should be balanced with a degree of individualisation, particularly in the care of medically complex patients. Furthermore, standardised processes should be evaluated frequently after implementation to assess for unintended consequences.
\end{abstract}

\section{INTRODUCTION}

Hepatic encephalopathy (HE) is a common complication of liver disease and patients with $\mathrm{HE}$ have high rates of healthcare utilisation. ${ }^{1}$ Several studies have looked at the delivery of quality care to hospitalised patients with cirrhosis and have concluded that a low proportion of patients receive high quality care. ${ }^{2-5}$ In 2010 , a set of quality indicators were derived by an expert panel with the intention of setting a platform for the delivery of quality care to patients with cirrhosis. ${ }^{6}$
Summary box

What is already known about this subject?

- Standardised order sets are being increasingly used in delivery of care in hospitalised patients, but data are limited on their impact on patients with cirrhosis

What are the new findings?

- The implementation of a standardised electronic health record order set for patients admitted with hepatic encephalopathy at our institution was associated with unexpected clinical outcomes including increased hospital length of stay and increased 30 day readmissions.

How might it impact on clinical practice in the foreseeable future?

- The results from our study illustrate the challenges in standardising the care of medically complex patients in general, where good clinical judgement is irreplaceable to ensure successful clinical outcomes.

Electronic health records (EHRs) are now almost ubiquitous in the American healthcare systems. Order sets are clinical decision support tools that aim to help providers by reducing variability, ordering pre-specified doses of medications and promoting adherence to guidelines in the care of patients, often who have a specific condition or disease. The use of order sets has been proposed as one approach to improve quality care in cirrhosis. ${ }^{5}$ Standardised order sets have been shown to improve adherence to clinical guidelines in venous thromboembolism and stroke, ${ }^{78}$ and improve clinical outcomes in the management of myocardial infarction and sepsis. ${ }^{910}$ One recent study looked at the development of an EHR order set to improve clinical outcomes in patients with variceal bleeding and demonstrated improvements in both the use and time to administration of antibiotics. ${ }^{11}$

Even though there is clear evidence of the benefits of order set use, there are also cautionary examples of their potential 
drawbacks on patient outcomes and healthcare utilisation. One study showed that a higher proportion of patients were exposed to increased risk of harm following the implementation of a standardised order set for venous thromboembolism prophylaxis. ${ }^{12}$ Overuse of medications such as sleeping aids, inappropriate longterm proton-pump inhibitor use and overuse of telemetry in the hospitalised setting have also been identified as unintended consequences of order set use. ${ }^{13-15}$

The effect of order set use on clinical outcomes on patients with cirrhosis and HE is unknown. We aimed to evaluate utilisation of an inpatient order set derived for use in patients with cirrhosis admitted to the hospital with $\mathrm{HE}$ and to evaluate the impact on clinical outcomes.

\section{METHODS}

\section{Setting}

The University of Minnesota Medical Center (UMMC) is a tertiary care medical centre with a high-volume liver transplant programme in the upper mid-western USA. Depending on patient acuity, patients with cirrhosis are admitted to either a Hospital Medicine team-staffed by a hospitalist with or without internal medicine residents-or the intensive care unit (ICU). An EHR (EPIC, Verona, Wisconsin, USA) was implemented at UMMC for use in the inpatient setting in 2011.

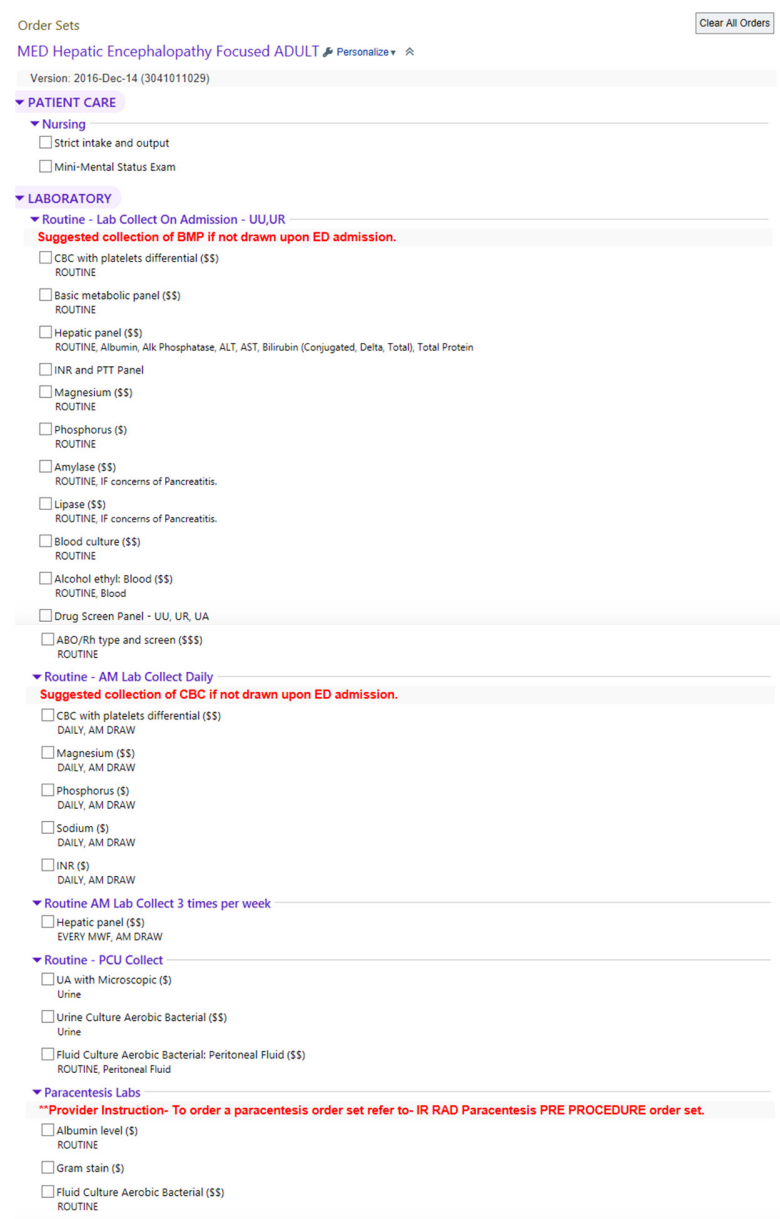

The HE order set was developed and made available for use at UMMC in December 2013. It is available (and encouraged) for use by all providers at any time during an admission and incorporates standardised pre-checked orders for the management of HE including a nursedriven lactulose protocol, which involves nursing assessment of a patient's mental status based on West Haven criteria. ${ }^{16}$ The order set includes a brief summary of stages of $\mathrm{HE}$ and frequent nursing assessment of HE severity. The presence of HE results in administration of $20 \mathrm{~g}$ lactulose orally, or $100 \mathrm{~g}$ lactulose rectally, every 2 hours as needed until resolution of symptoms. The content of this order set is consistent with evidence-based, standard practices in the management of HE. ${ }^{16}$

In addition, the order set also includes pre-checked orders for laboratory and medication orders for rifaximin, as well as options to order radiology studies and electrolyte replacement (figure 1). Of note, this order set was developed and implemented prior to conceptualisation and conduction of this study. This study was conducted with the approval of the University of Minnesota Institutional Review Board.

\section{Study design}

We performed a single centre, retrospective review of EHR data of the patients with cirrhosis admitted to

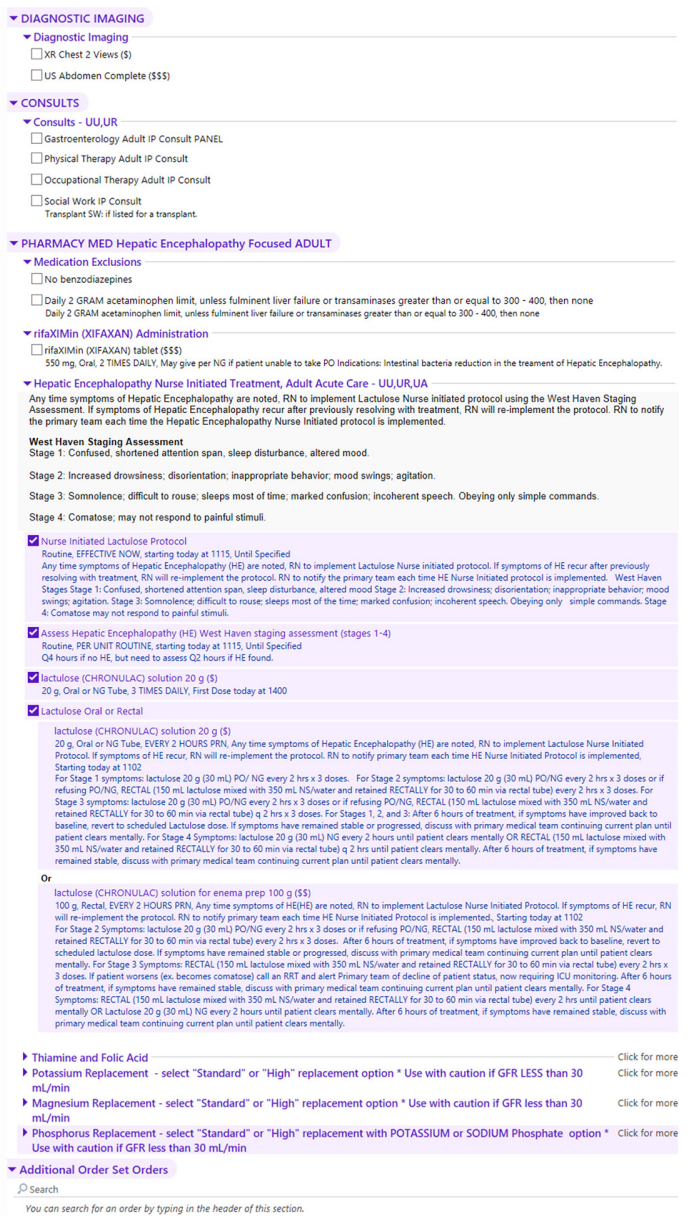


Table 1 Baseline characteristics

\begin{tabular}{|c|c|c|c|}
\hline & $\begin{array}{l}\text { Order-set used } \\
n=263\end{array}$ & $\begin{array}{l}\text { Order-set not used } \\
n=78\end{array}$ & $P$ value \\
\hline Female, n (\%) & $108(41)$ & $27(35)$ & 0.31 \\
\hline Caucasian race, $\mathrm{n}(\%)$ & $204(84)$ & $64(86)$ & 0.55 \\
\hline Age, mean (SD) & $56.0(10.5)$ & $58.9(11.3)$ & 0.05 \\
\hline MELD, mean (SD) & $20.7(8.5)$ & $21.0(7.2)$ & 0.79 \\
\hline MELD-Na, mean (SD) & $19.7(10.2)$ & $21.3(8.4 \%)$ & 0.20 \\
\hline Aetiology of liver disease & & & 0.22 \\
\hline Alcohol, n (\%) & $113(45)$ & $27(35)$ & \\
\hline Hepatitis C, n (\%) & $28(11)$ & $14(18)$ & \\
\hline Alcohol and hepatitis C, n (\%) & $28(11)$ & $7(9)$ & \\
\hline Other, n (\%) & $81(32)$ & $29(38)$ & \\
\hline \multicolumn{4}{|l|}{ Comorbid conditions } \\
\hline CKD, n (\%) & $60(23)$ & $12(15)$ & 0.16 \\
\hline ESRD, n (\%) & $14(5)$ & $4(5)$ & 1.00 \\
\hline HIV, n (\%) & $1(0.4)$ & $1(1)$ & 0.41 \\
\hline Diabetes, n (\%) & $104(40)$ & $27(35)$ & 0.43 \\
\hline COPD, n (\%) & $20(8)$ & $8(10)$ & 0.45 \\
\hline Coronary artery disease, n (\%) & $21(8)$ & $7(9)$ & 0.78 \\
\hline No. of hospital encounters, $n$ & 736 & 244 & \\
\hline Rifaximin use, n (\%) & $712(97)$ & $203(83)$ & $<0.001$ \\
\hline Precipitant of HE & & & 0.13 \\
\hline Medication non-adherence, n (\%) & $247(34)$ & $80(33)$ & \\
\hline Unknown precipitant, n (\%) & $128(17)$ & $64(23)$ & \\
\hline GI bleed, n (\%) & $33(4)$ & $13(5)$ & \\
\hline Urinary tract infection, n (\%) & $76(10)$ & $13(5)$ & \\
\hline Spontaneous bacterial peritonitis, n (\%) & $44(6)$ & $13(5)$ & \\
\hline Other, n (\%) & $208(28)$ & $70(29)$ & \\
\hline
\end{tabular}

CKD, chronic kidney disease; COPD, chronic obstructive pulmonary disease; ESRD, end-stage renal disease; GI, Gastrointestinal; HE, hepatic encephalopathy; MELD, model of end-stage liver disease.

UMMC with HE from December 2013 to December 2017. Hospital admissions for which the EHR order set was used at hospital admission were compared with admissions where the order set was not used.

\section{Study outcomes}

The primary outcome was hospital length of stay (LOS). Secondary outcomes were 30-day readmissions, in-hospital complications, in-hospital mortality and 90-day mortality. In-hospital complications included hypernatraemia (defined as serum sodium $>145 \mathrm{mEq} / \mathrm{L}$ or an increase in serum sodium $>5 \mathrm{mEq} / \mathrm{L}$ at any point in the hospital admission); hypokalaemia (defined as serum potassium $<3.5 \mathrm{mEq} / \mathrm{L}$ at any point in the admission) and acute kidney injury (defined as an increase in serum creatinine $>0.3 \mathrm{mg} / \mathrm{dL}$ over 48 hours).

\section{Study population}

Patients were included in the study if they were admitted to the UMMC Hospital Medicine service with an International Classification of Diseases-Ninth Revision (ICD-9) or ICD-10 code for cirrhosis and HE or altered mental status in admission or discharge coding. All admissions for $\mathrm{HE}$ were validated by manual chart review by a single physician in accordance with the definition of $\mathrm{HE}$ provided in the current American Association for the Study of Liver Diseases practice guideline. ${ }^{16}$ Patients with a diagnosis code for HE that did not meet criteria for HE after chart review were excluded from this study.

Patients were also excluded if they were less than 18 years old; if they had previously received a liver transplant; if they were originally admitted for non-HE condition; if they were admitted to the ICU or any other service than Hospital Medicine; if they were admitted for another acute neurological condition (cerebrovascular accident, meningitis, acute intoxication, intracranial haemorrhage or seizure) or if they were placed on comfort care/ hospice care during their hospitalisation. 


\section{Data acquisition}

Patient demographics (age, gender, ethnicity); hospitalisation data (admission and discharge dates, hospital readmission data, hospital admission unit); laboratory values; medications; co-morbid conditions and order-set utilisation data were obtained from the University of Minnesota Clinical and Translational Science Institute's clinical data repository via their Informatics Consulting Service. Aetiology of liver disease and precipitating factor for HE was obtained by manual chart review by a single physician.

\section{Statistical analysis}

Descriptive statistics were calculated and presented by group. Mean and SD were presented for continuous variables that are normally distributed. Median and IQR were presented for length of stay and number of readmissions as their distribution was skewed. Frequency and percentage were presented for categorical variables.

Bivariate analysis was conducted to compare order set use versus standard care using two-sample t-test for continuous variables that were normally distributed, nonparametric Wilcoxon test for length of stay and number of readmissions and $\chi^{2}$ test (or Fisher's exact test if any cell count was less than 5) for categorical variables. Multivariate analysis was performed to examine the effect of order set use on patient outcomes, adjusting for patient demographics including age at first HE episode, gender, race, aetiology of liver disease, rifaximin use and precipitant for HE. These covariates were selected based on scientific rationale as clinical factors known to affect clinical outcomes in patients with HE. Negative binomial regression models were conducted for length of stay and number of readmissions, and incidence rate ratio (IRR) was presented. Logistic regression model was conducted for binary outcomes, and OR was presented. Analyses were performed in SAS V.9.4 (SAS Institute). P values $<0.05$ were considered statistically significant.
Table 3 Adjusted risk for clinical outcomes with regard to order set use

\begin{tabular}{|c|c|c|}
\hline & & $P$ value \\
\hline $\begin{array}{l}\text { Hospital length of } \\
\text { stay }\end{array}$ & IRR 1.37 (95\% $\mathrm{Cl} 1.20$ to 1.57$)$ & $<0.001$ \\
\hline $\begin{array}{l}\text { 30-day } \\
\text { readmissions }\end{array}$ & OR 1.88 (95\% Cl 1.04 to 3.43$)$ & 0.04 \\
\hline \multicolumn{3}{|l|}{ Complications } \\
\hline Hypernatraemia & OR 1.19 (95\% Cl 0.86 to 1.66$)$ & 0.11 \\
\hline Hypokalaemia & OR 1.72 (95\% Cl 1.22 to 2.43$)$ & 0.002 \\
\hline $\begin{array}{l}\text { Acute kidney } \\
\text { injury }\end{array}$ & OR 1.31 (95\% Cl 0.94 to 1.85$)$ & 0.11 \\
\hline 90-day mortality & OR 1.02 (95\% Cl 0.55 to 1.89$)$ & 0.95 \\
\hline
\end{tabular}

Outcomes adjusted for age, gender, race, aetiology of liver disease, rifaximin use and precipitant for hepatic encephalopathy.

IRR, incidence rate ratio.

\section{RESULTS}

\section{Baseline characteristics}

There were a total of 341 unique patients with 980 admissions over the study period: 263 unique patients with 736 admissions where the order set was implemented, and 78 unique patients with 244 admissions where the order set was not implemented. Patients in the group where the order set was not used were older compared with the group where the order set was used (mean age 58.9 years vs 56.0 years, $\mathrm{p}=0.05$ ). Alcohol-related cirrhosis was the most common aetiology of liver disease in both groups. The mean model of end-stage liver disease (MELD)/ MELD-Na was 20.9/20.5 for the entire cohort. No significant differences were observed between the two groups for gender, ethnicity, mean MELD/MELD-Na score, co-morbidities, aetiology of liver disease or precipitant of HE. Rifaximin was used in $97 \%$ of patient encounters when order set was used compared with $83 \%$ when the order set was not used, $\mathrm{p}<0.001$. The most common documented

Table 2 Unadjusted clinical outcomes comparing patients with and without order set use

\begin{tabular}{lccc}
\hline & $\begin{array}{l}\text { Order set used } \\
\mathbf{n = 2 6 3}\end{array}$ & $\begin{array}{l}\text { Order set not used } \\
\mathbf{n = 7 8}\end{array}$ & P value \\
\hline Total no. of admissions, $\mathrm{n}$ & 736 & 244 & $<0.001$ \\
\hline Length of stay, median (IQR) & $4(3-8)$ & $3(2-7)$ & 0.001 \\
\hline $\begin{array}{l}\text { No. of readmission within 30 days, median (IQR) } \\
\text { Readmitted within 30 days of previous discharge, } \mathrm{n}(\%)\end{array}$ & $148(56)$ & $0(0-1)$ & 0.01 \\
\hline Complications & & $31(40)$ & 0.05 \\
$\quad$ No. of encounters with hypernatraemia, $\mathrm{n}(\%)$ & $379(51)$ & $108(44)$ & 0.003 \\
\hline No. of encounters with hypokalaemia, $\mathrm{n}(\%)$ & $341(46)$ & $87(36)$ & 0.19 \\
\hline No. of encounters with AKI, $\mathrm{n}(\%)$ & $342(47)$ & $102(42)$ & 1 \\
\hline In-hospital mortality & $5(2 \%)$ & $1(1 \%)$ & 0.34 \\
\hline 90-day mortality & $93(35 \%)$ & $23(29 \%)$ & \\
\hline
\end{tabular}

$\mathrm{AKI}$, acute kidney injury. 
HE precipitant was medication non-adherence. Despite extensive manual chart review, a large number of admissions had an unknown precipitant (table 1).

\section{Primary outcome}

The median LOS for admissions where the order set was used was 4 days (IQR 3-8 days) compared with 3 days (IQR 2-7 days) for admissions where the order set was not used $(p<0.001)$. After adjustment for age, gender, race, aetiology of liver disease, rifaximin use and HE precipitant, the incidence rate for increased LOS for the order set use group was $37 \%$ higher than the incidence rate for the group which did not use the order set (IRR 1.37 (95\% CI 1.20 to 1.57 ), $\mathrm{p}<0.001$ ) (tables 2 and 3 ).

\section{Secondary outcomes}

Thirty-day readmissions were more common in the order set group, $56 \%$ vs $40 \%, \mathrm{p}=0.01$. The median number of readmissions was 1 (IQR 0-3) for admissions where the order set was used compared with 0 (IQR $0-1$ ) when the order set was not used $(\mathrm{p}=0.001)$. After adjustment for age, gender, race, aetiology of liver disease, rifaximin use and HE precipitant, the OR for readmission in patients within 30 days for whom the order set was used was 1.88 (95\% CI 1.04 to 3.43), $\mathrm{p}=0.04$ (tables 2 and 3 ).

With regard to in-hospital complications, hypernatraemia occurred in $51 \%$ of admissions where the order set was used compared with $44 \%$ when the order set was not used, $\mathrm{p}=0.05$; OR 1.19 (95\% CI 0.86 to 1.66 ), $\mathrm{p}=0.11$, on multivariate analysis. Hypokalaemia occurred in $46 \%$ of admissions using the order set compared with $36 \%$ of admissions that did not use the order set, $\mathrm{p}=0.003$; OR $1.72(95 \%$ CI 1.22 to 2.43$), \mathrm{p}=0.002$, on multivariate analysis. Acute kidney injury did not vary depending on order set usage, $47 \%$ vs $42 \%, \mathrm{p}=0.19$; OR 1.31 (95\% CI 0.94 to 1.85 ), $\mathrm{p}=0.11$, on multivariate analysis (tables 2 and 3 ).

In-hospital mortality was $2 \%$ for admissions using the order set compared with $1 \%$ for those not using the order set but this did not reach statistical significance, $\mathrm{p}=1$. Multivariate analysis was unable to be performed for in-hospital mortality due to the low number of deaths. Ninety-day mortality was $35 \%$ for admissions using the order set compared with $29 \%$ for admissions not using the order set, $\mathrm{p}=0.34$; OR 1.02 (95\% CI 0.55 to 1.89 ), $\mathrm{p}=0.95$, on multivariate analysis (tables 2 and 3 ).

\section{DISCUSSION}

HE is a common complication of cirrhosis for which hospital admissions are increasing, making it an ideal target for the implementation of a standardised order set. ${ }^{17}$ However, the pathogenesis of HE is complex and remains poorly understood-and in our study, poorly documented. HE is a syndrome with several causes including infection and medication non-adherence; a recent study showed that $40 \%-76 \%$ of patients admitted with HE had multiple concurrent precipitants. ${ }^{18}$ With the fragility of patients with decompensated cirrhosis in general, careful assessment and management of all patients admitted to hospital with HE is essential.

Standardised EHR order sets have been proposed as one solution to address the current deficit in quality care delivered to patients with cirrhosis. ${ }^{2-5}$ In our study, despite high utilisation of an inpatient EHR order set for $\mathrm{HE}$, order set use was associated with increased hospital LOS and 30-day readmissions in this study. These findings persisted on multivariate analysis and demonstrate the difficulty in adopting standardised order sets in the care of medically complex patients. It does not appear that patients for whom providers opted to use the order set were significantly clinically different than those patients in whom the order set was not used.

Adherence to standardised order sets can have unintended outcomes in patient care. ${ }^{12-15} 19$ Our findings contribute to an emerging body of literature describing the limitations of order set use and demonstrate the importance of critical thinking in patient care, particularly in a patient population as susceptible to harm as those with cirrhosis. ${ }^{20} \mathrm{HE}$ is a syndrome-not a single entity-and careful, expert attention to the underlying precipitants as well as the recognition of the potential adverse effects of any intervention is fundamental. Certainly, medication non-adherence is a common precipitant-and lactulose is required in majority of patients admitted with HE. However, a diagnostic and treatment approach that lacks nuanced tailoring to a specific patient's presentation can be problematic. While order sets can reduce unnecessary variation in care, a degree of individualisation may be necessary in the management of these complex patients; in fact, order sets could be modified to encourage such assessment and individualisation.

One possible explanation for our findings is that the increased rate of complications observed in the order set group would logically lead to both increased hospital LOS and 30-day readmissions. Order set use was significantly associated with hypokalaemia—a known side effect of lactulose overuse-which may appear innocuous at first glance. However, hypokalaemia has been shown elsewhere to increase hospital and ICU lengths of stay in patients with HE. ${ }^{21}$ Moreover, hypokalaemia is associated with increased all-cause mortality in the elderly and in patients with chronic kidney disease (CKD) ${ }^{22}{ }^{23}$ We also observed trends for increased rates of hypernatremia and acute kidney injury, both of which are more traditionally associated with poor clinical outcomes in patients with cirrhosis. ${ }^{2425}$ There are multiple possible explanations for these findings, but we posit that patients treated with the order set were treated more aggressively with lactulose. Overuse of diuretics may have contributed to the increased rates of hypokalaemia and acute kidney injury seen in the order set group-and indeed may even account for a significant proportion of the 'unknown' HE precipitants in the whole cohort-but would not explain the higher rates of hypernatraemia. Administration of lactulose is the foundation of the treatment of HE but can have significant side effects including hypovolaemia, 
hypokalaemia and metabolic acidosis. It is possible that our order set was too aggressive in the administration of lactulose, particularly given the high rate of comorbidities (especially CKD and diabetes) in our patient population. Our outcomes may also have been a reflection of the high mean MELD-Na score at admission in our cohort, which in itself has been associated with higher readmission rates, although a significant difference was not observed between the two study groups. ${ }^{26-28}$

On a positive note, we observed higher rates of rifaximin use in the order set group. Although not a specific outcome of our study, rates of rifaximin use were high in both groups but significantly higher in the order set group. Rifaximin is a non-absorbable antibiotic that has been shown to reduce the risk of development of $\mathrm{HE}$ and improves quality of life outcomes in patients with cirrhosis. ${ }^{29}{ }^{30}$ In a recent metaanalysis, the use of rifaximin was associated with reduced overall mortality in patients with HE. ${ }^{31}$ Despite increased rifaximin use, we did not observe any improvement in mortality in the order set group: this may again be a reflection of higher baseline MELD-Na in our cohort but may also be due to the exclusion of patients admitted to the ICU from our study.

As our study groups were not randomised, it is important to consider why the order set was used in some encounters and not others. Both groups were similar with the exception of slightly older age in the order set group, which was not large enough to account for the difference in outcomes. Reasons for non-utilisation of order sets may include provider preference, lack of awareness of order set and lack of mandatory implementation within a health system. ${ }^{32} 33$ In this study, it is possible that use of the order set was affected by providers' perceived loss of control with regard to the nurse-driven lactulose protocol. The remainder of the content of the order set was consistent with standard, evidence-based practices and was unlikely to have contributed to these concerns.

Our study is the first to evaluate the implementation of an EHR order set in patients admitted to hospital with HE. Our findings are important due to their unexpected nature and illustrate the difficulty encountered in improving the quality of care in patients with decompensated cirrhosis. Hospital length of stay and 30-day readmissions are well-described metrics for resource utilisation which have significant financial implications. ${ }^{34} 35$ Increased hospital length of stay and readmissions have been shown both to increase the cost of care and worsen clinical outcomes in patients with decompensated cirrhosis. ${ }^{26}{ }^{36}$ This is particularly relevant at a time when healthcare costs are spiralling out of control in the USA: hospitalisation costs for patients with cirrhosis increased by $30 \%$ to $\$ 7.37$ billion in $2014 .{ }^{37}$

The strengths of our study include a large number of patients and patient encounters. Manual review of our data ensured accuracy and granularity, particularly with regard to HE precipitant which can be difficult to elucidate with automated data collection. The 4-year study period also accounted for any lag that can be occur following the implementation of EHR order set due to 'late adopters'. Limitations of our study include the single centre, retrospective design which may limit the generalisability of our results. Second, it can be difficult to capture all readmissions to a tertiary care referral hospital as patients often re-present to other institutions. Third, a large number of HE precipitants in our study were deemed 'unknown' despite extensive evaluation; however, this is a reflection of real-world practice. Finally, we acknowledge that patients with more severe $\mathrm{HE}$ at presentation may have preferentially had the order set used in their care. However, it is difficult to fully account for this possibility in our study: the West Haven criteria have significant intra-operator and inter-operator variability, and the mental status of patients with significant liver disease can fluctuate markedly. ${ }^{38}$ Again, we note that there was no significant difference in baseline liver function, represented by the MELD-Na score on admission, between the two study groups.

In conclusion, the implementation of a standardised EHR order set for patients admitted with HE at our institution was associated with unexpected clinical outcomes including increased hospital LOS and increased 30-day readmissions. The results from our study illustrate the challenges in standardising the care of medically complex patients in general, where good clinical judgement is irreplaceable to ensure successful clinical outcomes. Moreover, attempts to standardise care should be carefully monitored for adverse effects. Further study is required to understand why providers may opt out of using order sets and to optimise the utilisation of EHR to improve the quality of care delivered to patients with cirrhosis in hospital.

Contributors NL designed the study. MKC performed chart review. QW performed data analysis. NL, APJO, MKC drafted and edited manuscript.

Funding This research was supported by the National Institutes of Health's National Center for Advancing Translational Sciences, grant UL1TR002494.

Disclaimer The content is solely the responsibility of the authors and does not necessarily represent the official views of the National Institutes of Health's National Center for Advancing Translational Sciences.

Competing interests None declared.

Patient consent for publication Not required.

Provenance and peer review Not commissioned; externally peer reviewed.

Data availability statement Sharable data not available due to institutional restrictions.

Open access This is an open access article distributed in accordance with the Creative Commons Attribution Non Commercial (CC BY-NC 4.0) license, which permits others to distribute, remix, adapt, build upon this work non-commercially, and license their derivative works on different terms, provided the original work is properly cited, appropriate credit is given, any changes made indicated, and the use is non-commercial. See: http://creativecommons.org/licenses/by-nc/4.0/.

ORCID iDs

Mandip KC http://orcid.org/0000-0002-2993-8321

Nicholas Lim http://orcid.org/0000-0001-9740-8923

\section{REFERENCES}

1 Rakoski MO, McCammon RJ, Piette JD, et al. Burden of cirrhosis on older Americans and their families: analysis of the health and retirement study. Hepatology 2012;55:184-91. 
2 Kanwal F, Kramer JR, Buchanan P, et al. The quality of care provided to patients with cirrhosis and ascites in the Department of Veterans Affairs. Gastroenterology 2012;143:70-7.

3 Buchanan PM, Kramer JR, El-Serag HB, et al. The quality of care provided to patients with varices in the Department of Veterans Affairs. Am J Gastroenterol 2014;109:934-40.

4 Ghaoui R, Friderici J, Visintainer P, et al. Measurement of the quality of care of patients admitted with decompensated cirrhosis. Liver Int 2014;34:204-10.

5 Lim N, Lidofsky SD. Impact of physician specialty on quality care for patients hospitalized with decompensated cirrhosis. PLoS One 2015;10:e0123490.

6 Kanwal F, Kramer J, Asch SM, et al. An explicit quality indicator set for measurement of quality of care in patients with cirrhosis. Clin Gastroenterol Hepatol 2010;8:709-17.

7 Khoury L, Dangodara AA, Lee J-A, et al. Implementation of a mandated venous thromboembolism clinical order set improves venous thromboembolism core measures. Hosp Pract 2014;42:89-99.

8 Elder KG, Lemon SK, Costello TJ. Increasing compliance with national quality measures for stroke through use of a standard order set. Am J Health Syst Pharm 2015;72:S6-10.

9 Ballesca MA, LaGuardia JC, Lee PC, et al. An electronic order set for acute myocardial infarction is associated with improved patient outcomes through better adherence to clinical practice guidelines. $J$ Hosp Med 2014;9:155-61.

10 Thiel SW, Asghar MF, Micek ST, et al. Hospital-wide impact of a standardized order set for the management of bacteremic severe sepsis. Crit Care Med 2009;37:819-24

11 Mayorga CA, Rockey DC. Clinical utility of a standardized electronic order set for the management of acute upper gastrointestinal hemorrhage in patients with cirrhosis. Clin Gastroenterol Hepatol 2013;11:1342-8.

12 Khanna R, Vittinghoff E, Maselli J, et al. Unintended consequences of a standard admission order set on venous thromboembolism prophylaxis and patient outcomes. J Gen Intern Med 2012;27:318-24.

13 Kara A, Isaacs AN, Nisly SA. Prescriptions for bedtime sedatives after the introduction of a general admission order set at an academic health center: the potential and pitfalls of order sets. $J$ Patient Saf 2017;13:232-6.

14 Rubins D, Boxer R, Landman A, et al. Effect of default order set settings on telemetry ordering. J Am Med Inform Assoc 2019;26:1488-92.

15 Eid SM, Boueiz A, Paranji S, et al. Patterns and predictors of proton pump inhibitor overuse among academic and non-academic hospitalists. Intern Med 2010;49:2561-8.

16 Vilstrup H, Amodio P, Bajaj J, et al. Hepatic encephalopathy in chronic liver disease: 2014 practice guideline by the American association for the study of liver diseases and the European association for the study of the liver. Hepatology 2014;60:715-35.

17 Hirode G, Vittinghoff E, Wong RJ. Increasing burden of hepatic encephalopathy among hospitalized adults: an analysis of the 2010 2014 national inpatient sample. Dig Dis Sci 2019;64:1448-57.

18 Pantham G, Post A, Venkat D, et al. A new look at precipitants of overt hepatic encephalopathy in cirrhosis. Dig Dis Sci 2017;62:2166-73.

19 Gupta A, Das SR, Pandey A. $\beta$-Blockers in Myocardial Infarction: Issues With Standard Admission Order Sets. JAMA 2018;319:1269-70.
20 Koppel R, Metlay JP, Cohen A, et al. Role of computerized physician order entry systems in facilitating medication errors. JAMA 2005;293:1197-203.

21 Gaduputi V, Chandrala C, Abbas N, et al. Prognostic significance of hypokalemia in hepatic encephalopathy. Hepatogastroenterology 2014;61:1170-4.

22 Gilligan S, Raphael KL. Hyperkalemia and hypokalemia in CKD: prevalence, risk factors, and clinical outcomes. Adv Chronic Kidney Dis 2017;24:315-8.

23 Bardak S, Turgutalp K, Koyuncu MB, et al. Community-acquired hypokalemia in elderly patients: related factors and clinical outcomes. Int Urol Nephrol 2017;49:483-9.

24 Kim WR, Biggins SW, Kremers WK, et al. Hyponatremia and mortality among patients on the liver-transplant waiting list. $N$ Engl $J$ Med 2008;359:1018-26.

25 Wong F, O'Leary JG, Reddy KR, et al. Acute kidney injury in cirrhosis: baseline serum creatinine predicts patient outcomes. Am J Gastroenterol 2017;112:1103-10.

26 Volk ML, Tocco RS, Bazick J, et al. Hospital readmissions among patients with decompensated cirrhosis. Am J Gastroenterol 2012;107:247-52.

27 Orman ES, Ghabril M, Emmett TW, et al. Hospital readmissions in patients with cirrhosis: a systematic review. J Hosp Med 2018;13:490-5

28 Patel R, Poddar P, Choksi D, et al. Predictors of 1-month and 3-months Hospital readmissions in decompensated cirrhosis: a prospective study in a large Asian cohort. Ann Hepatol 2019;18:30-9.

29 Bass NM, Mullen KD, Sanyal A, et al. Rifaximin treatment in hepatic encephalopathy. N Engl J Med 2010;362:1071-81.

30 Sidhu SS, Goyal O, Mishra BP, et al. Rifaximin improves psychometric performance and health-related quality of life in patients with minimal hepatic encephalopathy (the RIME trial). Am J Gastroenterol 2011;106:307-16.

31 Kimer N, Krag A, Møller S, et al. Systematic review with metaanalysis: the effects of rifaximin in hepatic encephalopathy. Aliment Pharmacol Ther 2014;40:123-32.

32 Wong B, Mamdani MM, Yu CH. Computerized insulin order sets and glycemic control in hospitalized patients. Am J Med 2017;130:366 e1-e6.

33 Trujillo JM, Barsky EE, Greenwood BC, et al. Improving glycemic control in medical inpatients: a pilot study. J Hosp Med 2008;3:55-63.

34 Fine MJ, Pratt HM, Obrosky DS, et al. Relation between length of hospital stay and costs of care for patients with community-acquired pneumonia. Am J Med 2000;109:378-85

35 Upadhyay S, Stephenson AL, Smith DG. Readmission rates and their impact on hospital financial performance: a study of Washington hospitals. Inquiry 2019;56:46958019860386.

36 Flamm SL. Considerations for the cost-effective management of hepatic encephalopathy. Am J Manag Care 2018;24:S51-61.

37 Desai AP, Mohan P, Nokes B, et al. Increasing economic burden in hospitalized patients with cirrhosis: analysis of a national database. Clin Trans/ Gastroenterol 2019;10:e00062.

38 Bajaj JS, Cordoba J, Mullen KD, et al. Review article: the design of clinical trials in hepatic encephalopathy--an International Society for Hepatic Encephalopathy and Nitrogen Metabolism (ISHEN) consensus statement. Aliment Pharmacol Ther 2011;33:739-47. 